\title{
On The Ergodic Behaviour of Fuzzy Markov Chains
}

\author{
J.Earnest Lazarus PiriyaKumar ${ }^{1}$, V. Sreevinotha ${ }^{2}$, \\ ${ }^{1,2}$ Dept., of Mathematics, Tranquebar Bishop Manckkam Lutheran College, Porayar, South India.
}

\begin{abstract}
Stochastic stability of Markov chains has an almost complete theory and forms a foundation for several other general techniques. A fuzzy Markov system is proposed and describe both determined and random behavior of complex dynamic systems. In this paper we study the ergodic behavior of a fuzzy Markov chain, and Consequently their weak and strong ergodic behavior.
\end{abstract}

Keywords: Fuzzy Markov Chain, Fuzzy Transition Probability Matrix, Non-Stationary Markov chain.

\section{Introduction}

A fuzzy Markov system is proposed to describe both determined and random functioning of Complex dynamic systems. Most fuzzy logic applications are intended for Control and analytic purpose [5,4]. Another group of application is system state prediction[3] conventional fuzzy systems cannot operate with random phenomena.

Control processes in real life plants consist of determined and random elements. Stochastic processes can be described using a Markov modeling approach[2]. However, this approach allows simulation of a limited number of system states depending on state quantification. Furthermore the transition probability matrix must have large size to achieve high accuracy of modeling. This disadvantage can be avoided using a combination of Markov modeling with fuzzy logic.

In order to extend the application area of both techniques a fuzzy Markov modeling approach was proposed[1].

Therefore fuzzy Markov systems could be used for smooth non-linear approximation of a multidimensional probability density function. In case, a Markov model represents a fuzzy inference system with the transition probability matrix stored within the rule base.

Stochastic processes with a dynamic system can often be assumed to be stationary and ergodic. In this case the Markov chain is homogeneous and its dynamics are described by the transition probability matrix P. In this paper we study the ergodic behavior of fuzzy Markov chains and consequently the concepts weak ergodicity and strong ergodicity of fuzzy Markov chains.

\section{Fuzzy Markov Chain}

In this paper we proposed the set of possible limiting distributions for finite state Markov chain with fuzzy transition probabilities by which we mean a non-stationary Markov chain defined by the stochastic process.

$\{X(t) ; t=0,1,2 \ldots\}$ wtth transition probabilities $P_{i j}(t)=P\{X(t+1)=j \mid X(t)=i\} 1 \leq i, j \leq n$

Which satisfy the condition $\alpha_{\mathrm{ij}} \leq \mathrm{P}_{\mathrm{ij}}(\mathrm{t}) \leq \beta_{\mathrm{ij}}$ for each $\mathrm{t}=0,1,2, \ldots$ where $0 \leq \alpha_{\mathrm{ij}} \leq \beta_{\mathrm{ij}} \leq 1$

Let $\mathrm{S}=\left\{\mathrm{x} ; \mathrm{x}=\left(\mathrm{x}_{1}, \ldots \mathrm{x}_{\mathrm{n}}\right\}, \sum_{i=1}^{n} x_{i}=1 ; x \geq 0\right\}$ i.e. the set of all $\mathrm{n}$-dimensional probability vectors. The norm of a vector $\mathrm{x} \in R^{n}$ is defined by $\|x\|=\sum_{i=1}^{n}|x|$ and we topologize the closed subsets of the metric space (S, \|\|$)$ with the Hausdorff metric d defined by

$$
\begin{array}{r}
\delta(A, B)= \\
\mathrm{d}(\mathrm{A}, \mathrm{B})=\operatorname{Max}[\delta(A, B), \delta(B, a)]
\end{array}
$$

for any closed $\mathrm{A}, \mathrm{B} \subseteq S$. We also define

$$
\delta(A, B)=\max _{x \in A} \min _{y \in B}\|x-y\|
$$

$\mathrm{x}_{\mathrm{i}}(\mathrm{t})=\mathrm{P}_{\mathrm{r}}\{\mathrm{X}(\mathrm{t})=\mathrm{i}\}$

$f_{i}$, denote the fuzzy states of a Markov chain without loss of generality let $f_{r}$ denote the initial fuzzy state, $f_{s}$ denote the terminal fuzzy state and $\mathrm{f}_{\mathrm{j}}$ denote the inter mediate fuzzy state.

\section{Ergodic Coefficients of Fuzzy Matrix}

Definition:3.1 Let $\mathrm{P}$ be a fuzzy stochastic matrix the ergodic coeffecient of $\mathrm{P}$ denoted as $\alpha(\mathrm{P})$ is defined by

Where $\left[\mathrm{P}_{\mathrm{frfj}}-\mathrm{P}_{\mathrm{fsfj}}\right]^{+}=\max \left(0, \mathrm{P}_{\mathrm{frfj}}-\mathrm{P}_{\mathrm{fsfj}}\right)$.

$$
\alpha(P)=1-\sup _{f r, f s} \sum_{f j=1}^{\infty}\left[P_{f r f j}-P_{f s f j}\right]^{+}
$$


Theorem: 3.1 Let $\mathrm{P}$ be a fuzzy stochastic matrix, then

Proof: Let fr and fs be fixed,

$$
\alpha(P)=\underset{f r, f s}{\inf } \sum_{f j=1}^{\infty} \min \left(\left(P_{f r f j}, P_{f s f j}\right)\right.
$$

Since $\left(P_{f r f j}-P_{f s f j}\right)^{+}=\left[P_{f r f j}-\min \left(P_{f r f j}, P_{f s f j}\right)\right]$ and since $\quad \sum_{f j=1}^{\infty} P_{f r f j}=1$

We have

$$
\begin{gathered}
1-\sum_{f j=1}^{\infty}\left[P_{f r f j}-P_{f s f j}\right]^{+}=1-\sum_{f j=1}^{\infty}\left[P_{f r f j}-\min \left(P_{f r f j}, P_{f s f j}\right)\right] \\
=\sum_{f j=1}^{\infty} \min \left(P_{f r f j}, P_{f s f j}\right)
\end{gathered}
$$

Taking the infimum of both sides over fr,fs we get

$$
\begin{gathered}
\underset{f r, f s}{\inf } \sum_{f j=1}^{\infty} \min \left(P_{f r f j}, P_{f s f j}\right)={ }_{f r, f s}^{i n f}\left[1-\sum_{f j=1}^{\infty}\left(P_{f r f j}-P_{f s f j}\right)\right]^{+} \\
=1-\sup _{f r, f s} \sum_{f j=1}^{\infty}\left[P_{f r f j}-P_{f s f j}\right]^{+}
\end{gathered}
$$

It sometimes more convenient to use $1-\alpha(\mathrm{P})$ instead of $\alpha(\mathrm{P})$ itself. In view of this we define $\delta(\mathrm{P})=1-\alpha(\mathrm{P})$ and $\delta(\mathrm{P})$ the delta coefficient of $\mathrm{P}$.

therefore $\delta(\mathrm{P})=1-\underset{f r, f s}{\inf } \sum_{f j=1}^{\infty} \min \left(P_{f r f j}, P_{f s f j}\right)$

Theorem: 3.2 If $\mathrm{P}$ and $\mathrm{Q}$ are fuzzy stochastic matrices the $\delta(\mathrm{QP})<\delta(\mathrm{Q}) \delta(\mathrm{P})$.

Proof: In definition 3.1 we introduced the notation $\mathrm{a}^{+}=\max (0, \mathrm{a})$. If we introduce $\mathrm{a}^{-}$to denote $\max (0,-\mathrm{a})$ then we have $a=a^{+}-a^{-}$.

Employing this notation we see that for any two rows i and k of a fuzzy stochastic matrix Q we have

This is true since

$$
\sum_{f j=1}^{\infty}\left(q_{f r f j}-q_{f s f j}\right)^{+}-\sum_{f j=1}^{\infty}\left(q_{f r f j}-q_{f s f j}\right)^{-}
$$

$$
\begin{array}{cl}
\sum_{f j=1}^{\infty}\left(q_{f r f j}\right. & \left.-q_{f s f j}\right)^{+}-\sum_{f j=1}^{\infty}\left(q_{f r f j}-q_{f s f j}\right)^{-} \\
& =\sum_{f j=1}^{\infty}\left(q_{f r f j}-q_{f s f j}\right) \\
=1-1 & =0
\end{array}
$$

If we define

$\mathrm{QP}=\mathrm{R}=\left(\gamma_{\mathrm{frfj}}\right)$

Then $\delta(\mathrm{QP})=\delta(\mathrm{R})$

For the moment fix fr and fs and consider

$$
=\sup _{f r, f s} \sum_{f l=1}^{\infty}\left[\gamma_{f r f l}-\gamma_{f s f l}\right]^{+}
$$

$$
\sum_{f l=1}^{\infty}\left[\gamma_{f r f l}-\gamma_{f s f l}\right]^{+}=\sum_{f l=1}^{\infty}\left[\sum_{f j} q_{f r f j} P_{f j f l}-q_{f s f j} P_{f j f l}\right]^{+}
$$

Let $\mathrm{E}=\left\{1: \sum_{f j}\left(q_{f r f j}-q_{f s f j}\right) P_{f j f l}>0\right\}$

That is $\mathrm{E}$ denotes those columns 1 , for which the values $\gamma_{f r f l}-\gamma_{f s f l}$ is positive using the set E, (1.2) can be written

$$
\sum_{f l \in E} \sum_{f l=1}^{\infty}\left[q_{f r f j}-q_{f s f j}\right] P_{f j f l}
$$

The order summation can be interchanged using Funinis theorem so (1.3) is equal to

$$
\sum_{f l=1}^{\infty}\left[q_{f r f j}-q_{f s f j}\right] \sum_{f l \in E} P_{f j f l}=\sum_{f j=1}^{\infty}\left[\left(q_{f r f j}-q_{f s f j}\right)^{+}-\left(q_{f r f j}-q_{f s f j}\right)^{-}\right] \sum_{f l \in E} P_{f j f l}
$$




$$
=\sum_{f j=1}^{\infty}\left(q_{f r f j}-q_{f s f j}\right)^{+} \sum_{f l \in E} P_{f j f l}-\left(q_{f r f j}-q_{f s f j}\right)^{-} \sum_{f l \in E} P_{f j f l}
$$

Now since all the terms in this difference are non-negative the difference is made larger if the first term is increased and the second decreased. That is in place of $\sum_{l \in E} P_{f j f l}$ we substitute $\sup _{f j} \sum_{f l \in E} P_{f j f l}$ in the first term of the difference and

$i n f_{l \in E} \sum_{l \in E} P_{f j f l}$ in the second term, using the first that

We get

$$
\sum_{f j=1}^{\infty}\left(q_{f r f j}-q_{f s f j}\right)^{+}=\sum_{f j=1}^{\infty}\left(q_{f r f j}-q_{f s f j}\right)^{-}
$$

The last expression is simplified to

$$
\begin{aligned}
\sum_{f l=1}^{\infty}\left[\gamma_{f r f l}\right. & \left.-\gamma_{f s f l}\right]^{+} \leq \sum_{f j=1}^{\infty}\left(q_{f r f j}-q_{f s f j}\right)^{+}\left[\sup _{f j} \sum_{l \in E} P_{f j f l}-i n f_{f j} \sum_{l \in E} P_{f j f l}\right] \\
& =\sum_{f j=1}^{\infty}\left(q_{f r f j}-q_{f s f j}\right)^{+} \sup _{f j 1 f j 2} \sum_{l \in E}\left(P_{f j 1 f l}-P_{f j 2 f l}\right) \\
& <\sum_{f j=1}^{\infty}\left(q_{f r f j}-q_{f s f j}\right)^{+} \sup _{f j 1 f j 2} \sum_{l=1}^{\infty}\left(P_{f j 1 f l}-P_{f j 2 f l}\right)^{+}
\end{aligned}
$$

So taking the supremums of both sides over fr and fs we get

$$
\sum_{f j=1}^{\infty}\left(q_{f r f j}-q_{f s f j}\right)^{+} \delta(p)
$$

$$
\delta(Q P)<\delta(Q) \delta(P)
$$

Theorem: 3.3 For all matrices $\mathrm{A}$ and $\mathrm{B}$ the following inequality holds $\|A B\|<\|A\|$. $\|B\|$

Proof: The case where $\|A\|$ or $\|B\|$ is either zero or infinite, is easily done. Therefore assume that $0<\|A\|<$ $\infty$ and $0<\|B\|<\infty$. Note that the $(\mathrm{fr}, \mathrm{fj})$ th element of $\mathrm{AB}$ is given by

Then

$$
\sum_{f k=1}^{\infty} a_{f r f s}-b_{f s f j}
$$

By Funinis theorem the last expression is equal to

$$
\begin{aligned}
\|A B\| & =\sup _{f r} \sum_{f j=1}^{\infty} \sum_{f s=1}^{\infty} a_{f r f s}-b_{f s f j} \\
& <\sup _{f r} \sum_{f j=1}^{\infty} \sum_{f s=1}^{\infty} a_{f r f s} b_{f s f j}
\end{aligned}
$$

$$
\begin{aligned}
& \sup _{f r} \sum_{f_{s=1}}^{\infty} a_{f r f s} \sum_{f s=1}^{\infty} b_{f s f j} \\
<\sup _{f r} & \sum_{f s=1}^{\infty} a_{f r f s} \sup _{f s} \sum_{f j=1}^{\infty} b_{f s f j}
\end{aligned}
$$

$=\|A\| \cdot\|B\|$

\section{Weak Ergodicity}

In this section we give several theorems in which the ergodic coefficient can be used to determine whether a non-stationary Markov chain is weakly ergodic.

Definition: 4.1 A non-stationary Markov chain is called Weakly ergodic if for all $\mathrm{m} \mathrm{lim}_{f s \rightarrow \infty} \underset{f(0) g(0)}{s u} \| f^{(m, f s)}-$ $g^{(m, f s)} \|=0$

Where $\mathrm{f}^{(0)}$ and $\mathrm{g}^{(0)}$ are starting vectors.

Theorem: 4.1 A non-stationary fuzzy Markov chain is weakly Ergodic if and only if for all $\mathrm{m}$

$$
\delta\left(P^{(m, f s)}\right) \rightarrow 0 \text { as } f s \rightarrow \infty
$$

Proof: Assume that for all $\mathrm{m}, \delta\left(P^{(m, f s)}\right) \rightarrow 0$ as $f s \rightarrow \infty$. 
Let $\mathrm{f}^{(0)}$ and $\mathrm{g}^{(0)}$ be any two starting vectors and let $\mathrm{m}$ and $\mathrm{fs}$ be fixed. Define a fuzzy stochastic matrix $\mathrm{Q}$ such that the first row is $\mathrm{f}^{(0)}$ and the remaining row are $\mathrm{g}^{(0)}$.

Consider the matrix $\mathrm{QP}^{(\mathrm{m}, \mathrm{fs})}=\mathrm{R}$. The first row of the matrix $\mathrm{R}$ is $\mathrm{f}^{(\mathrm{m}, \mathrm{fs})}$ and the remaining rows are $\mathrm{g}^{(\mathrm{m}, \mathrm{fs})}$. Therefore since the value of $\delta(\mathrm{R})$ is determined by the rows of R we have

$$
\begin{aligned}
& \delta\left(Q P^{(m, f s)}\right)=\delta(R) \\
= & \frac{1}{2} \sup _{f r f j} \sum_{f l=1}^{\infty} \gamma_{f r f l}-\gamma_{f j f l} \\
= & \frac{1}{2} \sup \sum_{f r j}^{\infty} f_{l}^{(m, f s)}-g_{l}^{(m, f s)} \\
= & \frac{1}{2}\left\|f_{l}^{(m, f s)}-g_{l}^{(m, f s)}\right\|
\end{aligned}
$$

Using theorem 3.2 and the fact that $\delta(\mathrm{Q})<1$, we note that

$$
\begin{gathered}
\left\|f_{l}^{(m, f s)}-g_{l}^{(m, f s)}\right\| \\
=2 \delta\left(Q P^{(m, f s)}\right) \\
\leq 2 \delta(Q) \delta\left(P^{(m, f s)}\right) \\
<2 \delta\left(P^{(m, f s)}\right)
\end{gathered}
$$

By assumption the right hand side goes to zero for each $\mathrm{m}$ as fs $\rightarrow \infty$. Further more it goes to zero independently if $\mathrm{f}^{(0)}$ and $\mathrm{g}^{(0)}$. So the chain is Weakly ergodic.

Conversely assume that for all $\mathrm{m}, \quad \underset{f(0) g(0)}{\sup }\left\|f^{(m, f s)}-g^{(m, f s)}\right\| \rightarrow 0$ as $k \rightarrow \infty$. Define $\mathrm{f}^{(0)}$ to be a starting vector with a one in the ith position and zero elsewhere and define $\mathrm{g}^{(0)}$ to be starting vector with a one in the jth position and zeros elsewhere.

Note that the vectors

Are the ith and jth rows of $p^{(m, f s)}$ respectively./ So

$$
\begin{gathered}
f^{(0)} p^{(m, f s)}=f^{(m, f s)} \text { and } \\
g^{(0)} p^{(m, f s)}=g^{(m, f s)}
\end{gathered}
$$

$$
\begin{aligned}
& \sum_{f l=1}^{\infty} P_{f r f l}(m, f s)-P_{f j f l}(m, f s) \\
& \quad=\left\|f_{\sup }^{(m, f s)}\right\| g^{(m, f s)} \| \\
& <\quad f(0) g(0)
\end{aligned}
$$

Since the Inequality holds for all $\mathrm{fr}$, fj it follows that

$$
\begin{aligned}
& 2 \delta\left(P^{(m, f s)}\right)=\sup _{f r g j} \sum_{f l=1}^{\infty} P_{f r f l}(m, f s)-P_{f j f l}(m, f s) \\
& <\sup _{f(0) g(0)}\left\|f^{(m, f s)}-g^{(m, f s)}\right\|
\end{aligned}
$$

And the last term tends to zero for all $\mathrm{m}$ as $\mathrm{fs} \rightarrow \infty$ by assumption.

Theorem: 4.2 Let $\{X n\}$ be a non-stationary fuzzy Markov chain with transition matrices $\{P n\}_{n=1}^{\infty}$. The chain $\{\mathrm{Xn}\}$ is weakly ergodic if and only if there exists a subdivision of $\mathrm{P}_{1}, \mathrm{P}_{2} \ldots$ In to blocks of matrices $\left[\mathrm{P}_{1} \mathrm{P}_{2} \mathrm{P}_{3} \ldots \mathrm{P}_{\mathrm{n}}\right]\left[\mathrm{P}_{\mathrm{n} 1+1} \mathrm{P}_{\mathrm{n} 2+1} \ldots . \mathrm{P}_{\mathrm{nj}+1}\right]$ such that

$\sum_{f l=0}^{\infty} \alpha\left(P^{(n j, n j+1)}\right)=\infty$ where $\mathrm{n}_{0}=0$.

Proof: The first part of the proof depends on the following result from analysis.

If $\{\epsilon j\}_{j=1}^{\infty}$ is a sequence of numbers with $0<\epsilon j<1$ for all $\mathrm{j}$ then the product $\left\{\prod\right\}_{n=1}^{\infty}\left(1-\epsilon_{j}\right)$ diverges to zero as $\mathrm{n} \rightarrow \infty$ if and only if

$\sum_{j=m}^{\infty} \epsilon_{j}=\infty$. If $\sum_{f l=0}^{\infty} \alpha\left(P^{(n f j, n f j+1)}\right)=\infty$ for all $i$.

$$
\begin{array}{lll}
\delta(\mathrm{P})=1- & \alpha(\mathrm{P}) & \text { we }
\end{array} \text { that }
$$

As $\mathrm{fl} \rightarrow \infty$.

Finally let $\mathrm{m}$ given and define $\mathrm{fr}=\min \left\{\mathrm{fj}: \mathrm{n}_{\mathrm{fj}}>\mathrm{m}\right\}$ and for $\mathrm{fs}>\mathrm{m}$ define $\mathrm{l}=\max \left\{\mathrm{fj}: \mathrm{n}_{\mathrm{fj}}<\mathrm{k}\right\}$ and note that $\mathrm{fl} \rightarrow \infty$ as $\mathrm{fs} \rightarrow \infty$. Then using (1) and theorem we 


$$
\delta\left(P^{(m, f s)}\right) \leq \delta\left(P^{(m, n f r)}\right) \prod_{f j=i}^{f l-1} \delta\left(P^{(n f j, n f j+1)}\right) \cdot \delta\left(P^{(n f j, n f j+1)}\right) \rightarrow 0 \quad \text { as } f s \rightarrow \infty
$$

Conversely assume that the chain is Weakly ergodic that is for all $\mathrm{m}$

$$
\delta\left(P^{(m, f s)}\right) \rightarrow 0 \quad \text { as } k \rightarrow \infty
$$

This implies that for all $\mathrm{m}$

$$
\alpha\left(P^{(m, f s)}\right) \rightarrow 1 \quad \text { as } k \rightarrow \infty
$$

Hence for $\mathrm{m}=0=\mathrm{n}_{0}$ there exists $\mathrm{n} 1$ such that $\alpha\left(P^{(0, n 1)}\right)>1 / 2$. Likewise given $\mathrm{n} 1$ there exists $\mathrm{n} 2>\mathrm{n} 1$ such that $\alpha\left(P^{(n 1, n 2)}\right)>1 / 2$

Proceeding this way we get

Which

$$
\sum_{f l=0}^{f s} \alpha\left(P^{(n f j, n f j+1)}\right)>\frac{f s+1}{2}
$$

$$
\text { diverges }
$$

Hence we have constructed a partition of the original sequence of matrices $\mathrm{p} 1, \mathrm{p} 2, \ldots$. In to blocks satisfying

$$
\sum_{f l=0}^{\infty} \alpha\left(P^{(n f j, n f j+1)}\right)=\infty
$$

\section{Strong Ergodicity}

In this section we present some theorems that give sufficient conditions for a chain to be strongly ergodic.

Theorem: 5.1 A non- stationary fuzzy Markov chain is strongly ergodic if and only if there is a sequence of constant fuzzy stochastic matrices $\left\{Q_{m}\right\}$ and for each $m$, there is a sequence of constant stochastic matrices $\left\{Q_{\mathrm{mk}}\right\}$ such that

$$
\begin{aligned}
& \text { (i) } \lim _{f s \rightarrow \infty}\left\|P^{(m, f s)}-Q_{m f s}\right\|=0 \text { and } \\
& \text { (ii) } \lim _{f s \rightarrow \infty}\left\|Q_{m f s}-Q_{m}\right\|=0
\end{aligned}
$$

Proof: Assume sequence of constant matricas $\{\mathrm{Qm}\}$ and $\{\mathrm{Qmfs}\}$ satisfying conditions (i) and (ii) exist. Since

$$
\left\|P^{(m, f s)}-Q_{m}\right\|<\left\|P^{(m, f s)}-Q_{m f s}\right\|+\left\|Q_{m f s}-Q_{m}\right\|
$$

It follows that for all $\mathrm{m}$,

Clearly if $\mathrm{Qm}=0$ for all $\mathrm{m}$, then by theorem,

$$
\lim _{f s \rightarrow \infty}\left\|P^{(m, f s)}-Q_{m f s}\right\|=0
$$

A non stationary fuzzy Markov chain with transition matrices $\{p n\}$ is strongly ergodic if and only if there exists a constant matrix $Q$ such that for each $m$

The chain will be strongly ergodic.

$$
\lim _{f s \rightarrow \infty}\left\|P^{(m, f s)}-Q\right\|=0
$$

In other words, it suffices to show that Qm is the same constant matrix for all $\mathrm{m}$. It is easy to show that $\mathrm{PmQm}=\mathrm{Qm}$

We also know from theorem that for any two matrices $\mathrm{A}$ and $\mathrm{B}$,

$\|A B\|<\|A\| .\|B\|$ hence we get

$$
\begin{aligned}
\| Q_{m-1}-Q_{m} & \|<\| Q_{m-1}-P^{(m, f s)}\|+\| P_{m} P^{(m, f s)}-P_{m} Q_{m}\|+\| P_{m} Q_{m}-Q_{m} \| \\
& =\left\|Q_{m-1}-P^{(m-1, f s)}\right\|+\| P_{m}\left(P^{(m, f s)}-Q_{m} \|\right. \\
& <\left\|Q_{m-1}-P^{(m-1, f s)}\right\|+\left\|P_{m}\right\| \|\left(P^{(m, f s)}-Q_{m} \|\right. \\
& <\left\|Q_{m-1}-P^{(m-1, f s)}\right\|+\|\left(P^{(m, f s)}-Q_{m} \|\right.
\end{aligned}
$$

By letting $k \rightarrow \infty$ we get $\left\|Q_{m-1}-Q_{m}\right\|=0$ which implies that $Q_{m-1}=Q_{m}$ for all $\mathrm{m}$.

Conversely of the chain is strongly ergodic then by setting $\mathrm{Qm}=\mathrm{Qmfs}=\mathrm{Q}$ for all $\mathrm{m}$ and fs it follows that (i) and (ii) are true.

Definition: 5.1 Let a be the class of stochastic matrices $P$ for which there exists at least one non-negative left eigen vector corresponding to the eigen value $\psi$ such that

$$
\|\psi\|=1
$$

Theorem: 5.2 Let $\{\mathrm{Pn}\}$ be a sequence of transition matrices corresponding to a non-stationary weakly ergodic Markov chain with $P n \in a$ for all $n$. If there exists a corresponding sequence of left eigen vectors $\psi$ n satisfying 
$\sum_{f j=1}^{\infty}\left\|\psi_{f j}-\psi_{f j+1}\right\|<\infty$

Then the chain is strongly ergodic.

Proof: The condition imposed on the left eigon vectors is stronger than assuming $\{\psi n\}_{n=1}^{\infty}$ converges in norm to some vector $\psi$. Hence we can define $\psi=\lim _{n \rightarrow \infty} \psi_{n}$ and note that $\left\|\psi_{n}-\psi\right\| \rightarrow 0$ as $n \rightarrow \infty$.

Since all of the $\psi_{n}$ 's have the property that their components are non-negative and add to one $\psi$ will also have this property.

Define $\mathrm{Q}$ to be the constant stochastic matrix with each row equal to $\psi$.

In order to show $\{\mathrm{Pn}\}$ is strongly ergodic it is sufficient to show $\|\left(P^{(m, f s)}-Q_{m} \| \rightarrow 0\right.$ as $k \rightarrow \infty$ for all $\mathrm{m}$.

For notational convenience let Qn denote the constant stochastic matrix with rows equal to $\psi_{n}$. Let $\mathrm{m}$ be fized using the triangle inequality and the fact that $P^{(m, f s)}=P^{(m, f l)} P^{(f l, f s)}$ we get

$$
\begin{gathered}
\left\|P^{(m, f s)}-Q\right\|<\left\|P^{(m, f s)}-Q_{f s}\right\|+\left\|Q_{f s}-Q\right\| \\
<\left\|P^{(m, f l)} P^{(f l, f s)}-Q_{f l+1} P^{(f l, f s)}\right\|+\left\|Q_{f l+1} P^{(f l, f s)}-Q_{f s}\right\|+\| Q_{f s}-Q
\end{gathered}
$$

In

$$
\text { order }
$$

prove

$$
\lim _{f s \rightarrow \infty}\left\|P^{\text {to }}, Q_{m s s}\right\|=0
$$

that

We let $\epsilon>0$ be given and show that there exists $\mathrm{k}$ such that for all fs $>\mathrm{k}\left\|P^{(m, f s)}-Q\right\|<\epsilon$ we do this by making each of the three terms on the right hand side of (3) less than $\in / 3$

We first consider the middle term of the right hand side of (3) and note that since $Q_{f l+1} P^{(f l, f s)}=Q_{f l+1}$ we have

$$
\begin{gathered}
Q_{f l+1} P^{(f l, f s)}=Q_{f l+1} P^{(f l+1, f s)} \\
=Q_{f l+1} P^{(f l+1, f s)}-Q_{f l+2} P^{(f l+1, f s)}+Q_{f l+2} P^{(f l+1, f s)} \\
=\left(Q_{f l+1}-Q_{f l+2}\right) P^{(f l+1, f s)}+Q_{f l+2} P^{(f l+1, f s)}
\end{gathered}
$$

Repeating this procedure on $Q_{f l+2} P^{(f l+1, f s)}$ we get

$$
Q_{f l+1} P^{(f l, f s)}=\left(Q_{f l+1}-Q_{f l+2}\right) P^{(f l+1, f s)}+\left(Q_{f l+2}-Q_{f l+3}\right) P^{(f l+2, f s)}+Q_{k}
$$

Hence using the triangle inequality theorem and the fact that $\delta\left(P^{(f j, f s)}\right)<1$ we get

$$
\begin{gathered}
\left\|Q_{f l+1} P^{(f l, f s)}-Q_{f s}\right\|=\left\|\sum_{j=f l+1}^{f s-1}\left(Q_{f j}-Q_{f j+1}\right) P^{(f l, f s)}\right\| \\
<\sum_{j=f l+1}^{f s-1}\left\|\left(Q_{f j}-Q_{f j+1}\right) P^{(f j, f s)}\right\| \\
<\sum_{j=f l+1}^{f s-1}\left\|\left(Q_{f j}-Q_{f j+1}\right)\right\| \delta P^{(f j, f s)} \\
<\sum_{j=f l+1}^{f s-1}\left\|\left(Q_{f j}-Q_{f j+1}\right)\right\|
\end{gathered}
$$

Since by construction Qfj has all its rows equal to $\psi_{f j}$, it follows that

Hence using assumption (2) we can choose

$$
\left\|\left(Q_{f j}-Q_{f j+1}\right)\right\|=\left\|\left(\psi_{f j}-\psi_{f j+1}\right)\right\|
$$

$\mathrm{fl} *>\mathrm{m}$ such that for all $\mathrm{k}>\mathrm{fl}{ }^{*}$

$$
\begin{gathered}
\left\|Q_{f l *+1} P^{(f l * f s)}-Q_{f s}\right\|=\left\|\sum_{j=f l *+1}^{f_{s}-1}\left(Q_{f j}-Q_{f j+1}\right)\right\| \\
=\left\|\sum_{j=f l *+1}^{f_{s-1}}\left(\psi_{f j}-\psi_{f j+1}\right)\right\|
\end{gathered}
$$

$<\in / 3$

With fl* fixed, next consider the first term of the right hard side of eigen vector. Since $\mathrm{P}^{\left(\mathrm{m}, \mathrm{fl}^{*}\right)}$ and $\mathrm{Q}_{\mathrm{fl}} \mathrm{f}^{+1}$ are stochastic matrices it follows that

So by the theorem

$$
\left\|P^{(f l * f s)}-Q_{f l *+1}\right\|<2
$$

$$
\begin{gathered}
\left\|P^{(m, f l *)} P^{(f l *, k)}-Q_{f l *+1} P^{(f l * f s)}\right\| \leq\left\|P^{(m, f l *)}-Q_{f l *+1}\right\| \delta P^{(f l * f s)} \\
\leq 2 \delta P^{(f l *, f s)}
\end{gathered}
$$

Using the assumption that the chain is weakly ergodic we can find $\mathrm{k} 1>\mathrm{fl} *$ such that for all $\mathrm{fs}>\mathrm{k} 1$ 
For such values of fs

$$
\delta P^{(f l *, f s)}<\in / 6
$$

$$
\left\|P^{(m, f l *)} P^{(f l *, k)}-Q_{f l *+1} P^{(f l *, f s)}\right\|<\epsilon / 3
$$

For the third term on the right hand side of eigen vector we note that $\psi_{k}$ converges in norm to $\psi$ and so

Here there exist $\mathrm{k} 2$ such that for all fs $>\mathrm{k} 2$ we have

$$
\log _{f s \rightarrow \infty}\left\|Q_{f s}-Q\right\|=0
$$

$$
\left\|Q_{f s}-Q\right\|<\epsilon / 3
$$

Therefore for

$\mathrm{Fs}>\max (\mathrm{k} 1, \mathrm{k} 2)$ we have

$\left\|P^{(m, f s)}-Q\right\|<\frac{\epsilon}{3}+\frac{\epsilon}{3}+\frac{\epsilon}{3}=\epsilon$.

\section{References}

[1]. V.Y.Arkov, T.V.Breikin and G.G.Kulikov, fuzzy Markov simulation technique for product testing equipment, prepr. ${ }^{\text {th }}$ FFAC workshop on intelligent Manufacturing systems IMS97 1997,415-419.

[2]. T.V.Breikin,V.Y.Arkov and G.G.Kulikov on stochastic system identification. Markov models approach, Proc. $2^{\text {nd }}$ Asian control Conf. ASCC97,v2,1997,775,778.

[3]. J.S.R.Jang and C.T.Sun, Predicting chaotic time series with fuzzy if-then rules, Proc.IEEE.Int.Conf. on fuzzy systems, San Francisco, 1993.

[4]. E.H.Mamdini, Advanced in linguistic synthesis with a fuzzy logic controller, Int. J.Man-Machine studies 1976,8,669-678

[5]. L.A.Zadeh, Outline of a new approach to the analysis of Complex systems and decision process, IEEE Trans on Systems Man and Cybernetics, 1973,3(1),28-44 